NASA Technical Memorandum 105947

106056

\title{
A Time-Accurate High-Resolution TVD Scheme for Solving the Navier-Stokes Equations
}

Hyun Dae Kim and Nan-Suey Liu

Lewis Research Center

Cleveland, Ohio

December 1992 


\title{
A Time-Accurate High-Resolution TVD Scheme for Solving the Navier-Stokes Equations
}

Hyun Dae Kim and Nan-Suey Liu

\author{
National Aeronautics and Space Administration \\ Lewis Research Center \\ Cleveland, Ohio 44135
}

\begin{abstract}
A TVD scheme has been developed and incorporated into an existing timeaccurate high-resolution Navier-Stokes code. The accuracy and the robustness of the resulting solution procedure have been assessed by performing many calculations in four different areas: shock tube flows, regular shock reflection, supersonic boundary layer, and shock boundary layer interactions. These numerical results compare well with corresponding exact solutions or experimental data.
\end{abstract}




\section{Introduction}

Recently, an iterative-implicit diagonally dominant factorization algorithm, together with a high order finite-difference scheme, for solving the multidimensional compressible unsteady Navier-Stokes equations has been developed. The important features of this solution algorithm, the finite difference scheme and some validated results were reported in Ref. [1]. The present work is a continuing effort at developing its shock-capturing capability through the use of flux limiters. A brief description of the resulting TVD (total variation diminishing) scheme is given in Section 2.

Standard test cases have been carried out to assess the overall accuracy of the current code, which embodies the solution algorithm/scheme presented in Ref. [1] and the shock-capturing capability developed under the current effort. In Section 3, four different shock-tube flows are calculated to demonstrate its capability of accurately tracking transient flow discontinuities including shock waves and contact discontinuities. In Section 4, the results of an oblique shock wave reflection are shown and compared with exact solutions. This demonstrates its accuracy in computing shocks that are not aligned with grid lines. To evaluate the possible effects of flux limiters on the numerical solution of flows containing boundary layers, calculations of a supersonic laminar boundary layer flow have been performed, the results are presented in Section 5. Using this viscous flow over

a flat plate as the starting condition, an oblique shock wave is then introduced to examine the interaction of an oblique shock wave with a laminar boundary layer. Both the transient development of the interaction and the comparison of the steadystate solution between the current calculation and the corresponding experiment are discussed in Section 6. 


\section{The Solution Algorithm and The TVD Scheme}

A relatively detailed discussion of the overall solution algorithm and a high resolution finite-difference scheme for the inviscid fluxes, termed as the FCTD scheme, can be found in Ref. [1]. In short, the basis of the solution algorithm is a diagonally dominant approximate factorization procedure. The factorization error and the timewise linearization error associated with this baseline procedure are reduced by performing Newton-type inner iterations at each time step. The robustness of the overall algorithm is enhanced by carrying out the temporal iterations in pairs to enforce the operational symmetry of the factorization procedure. The temporal accuracy is increased to second-order by using three-point backward time differencing. The viscous fluxes are evaluated by using the halfspacing second-order central differencing scheme. The inviscid fluxes are evaluated by the so called FCTD scheme, which is an amended fourth-order central differencing scheme with its injected numerical dissipation having the same form as the entire dissipative part of the truncation error intrinsic to the third-order-biased upwind scheme. Under the current effort, a TVD form of the FCTD scheme has been developed to capture flow discontinuities that often occur in many practical problems. A brief description of this development follows.

The two dimensional Navier-Stokes equations in generalized coordinates $(\xi, \eta)$ can be written as

$$
\frac{\partial \widehat{Q}}{\partial \tau}+\frac{\partial}{\partial \xi}\left(\widehat{E}-\widehat{E}_{v}\right)+\frac{\partial}{\partial \eta}\left(\widehat{F}-\widehat{F}_{v}\right)=0
$$

where $\widehat{Q}=Q / J ; Q=(\rho, \rho u, \rho v, e)^{T}$, and $J=\xi_{\mathrm{x}} \eta_{\mathrm{y}}-\xi_{\mathrm{y}} \eta_{\mathrm{x}}$ is the metric Jacobian. Here, $\tau$ is the time; $\rho$ is the fluid density; $e$ is the total internal energy per unit volume; $u$ and $\mathrm{v}$ are the velocity components in the $\mathrm{x}$ and $\mathrm{y}$ directions of a Cartesian 
coordinates system. The transformed inviscid fluxes are denoted by $\widehat{E}$ and $\widehat{F}$ and the transformed viscous fluxes are denoted by $\widehat{E}_{\mathrm{v}}$ and $\widehat{\mathrm{F}}_{\mathrm{v}}$. The specific forms of these transformed fluxes are well known and will not be repeated here. Using an iterative implicit technique and three point backward time differencing, equation (1) leads to

$$
\left[\frac{\alpha}{\Delta \tau} \delta \hat{Q}+\frac{\partial}{\partial \xi}\left(\delta \widehat{E}-\delta \widehat{E}_{v}\right)+\frac{\partial}{\partial \eta}\left(\hat{\boldsymbol{F}}-\delta \widehat{F}_{v}\right)\right]^{(m)}=R H S^{(m)}
$$

where

$$
\begin{aligned}
R H S^{(m)}= & \frac{1}{\Delta \tau}\left[\alpha \sum_{l=1}^{m-1}(\delta \hat{Q})^{(l)}+(1-\alpha)(\delta \hat{Q})^{(n-1)}\right] \\
& -\left[\frac{\partial}{\partial \xi}\left(\widehat{E} \cdot \widehat{E}_{v}\right)+\frac{\partial}{\partial \eta}\left(\widehat{F} \cdot \widehat{F}_{v}\right)\right]^{(m)}
\end{aligned}
$$

and

$$
\begin{aligned}
& (\delta f)^{l}=f^{(l+1)}-f^{(l)} \quad l=1,2,3, \ldots m \\
& \alpha=1.5
\end{aligned}
$$

In the above equations, $f$ denotes an arbitrary quantity, $l$ represents an iteration index, and $m$ is an intermediate iteration level between the $n$-th and $(n+1)$-th time levels.

The construction of operators approximating the left hand side of Eq. (2) and the evaluation of the viscous fluxes in Eq. (3) are the same as those described in Ref. [1]. The development of a TVD scheme for evaluating the inviscid fluxes in Eq. (3) is based on the FCTD scheme described in Ref. [1]. Taking $\frac{\partial \widehat{\mathbf{E}}}{\partial \xi}$ in Eq. (3) as an example, and dropping ' $\wedge$ ' from the flux $\widehat{\mathbf{E}}$, the working formula for FCTD scheme is given by

$$
[\mathrm{FCTD}](\mathrm{E})_{\mathrm{i}}=(1-\beta)[\mathrm{FC}](\mathrm{E})_{\mathrm{i}}+\beta[\mathrm{TU}](\mathrm{E})_{\mathrm{i}}
$$


where $[\mathrm{FC}](\mathrm{E})_{\mathrm{i}}$ and $[\mathrm{TU}](\mathrm{E})_{\mathrm{i}}$ denote the fourth-order central differencing and the third-order-biased upwind differencing of $\frac{\partial E}{\partial \xi}$ at the spatial point $(i, j)$ respectively. It is noted here that, for convenience, the index $\mathrm{j}$ associated with the $\eta$-direction has been dropped in Eq. (4).

The numerical dissipation of the FCTD finite-difference scheme is essentially an infinite series with its elements being the fourth and higher even derivatives of the absolute fluxes. The relative amount of added dissipation can be controlled through an adjustable parameter $\beta$. When $\beta=0$, FCTD becomes the fourth-order central differencing scheme without numerical dissipation and when $\beta=1$, it becomes the third-order-biased upwind scheme.

Following the steps detailed in Ref. [2], a TVD form of the FCTD scheme can be constructed, and it has the following form :

$$
\frac{\partial \mathrm{E}}{\partial \xi}=\mathrm{E}_{\mathrm{i}+\frac{1}{2}}-\mathrm{E}_{\mathrm{i}-\frac{1}{2}} \quad(\Delta \xi=1)
$$

where

$$
E_{i+\frac{1}{2}}=\left[\frac{1}{2}\left(E_{i+1}+E_{i}\right)-\frac{1}{2}\left(\Delta^{+} E_{i}^{+}-\Delta^{+} E_{i}\right)\right]+\left(\delta_{i}^{+}-\delta_{i+1}^{-}\right)
$$

and

$$
\begin{aligned}
& \begin{aligned}
\left(\delta_{i}^{+}-\delta_{i+1}^{-}\right) & =\frac{1}{6}(3-\beta)\left[\phi\left(\frac{1}{r_{i}^{+}}\right) \Delta^{+} E_{i}^{+}-\phi\left(\frac{1}{r_{i+1}}\right) \Delta^{+} E_{i}\right] \\
& -\frac{1}{12}\left[(1-\beta) \phi\left(\frac{1}{r_{i+1}^{+}}\right) \Delta^{+} E_{i+1}^{+}+(1+\beta) \phi\left(r_{i+1}\right) \Delta^{+} E_{i+1}^{-}\right] \\
& +\frac{1}{12}\left[(1+\beta) \phi\left(r_{i}^{+}\right) \Delta^{+} E_{i-1}^{+}+(1-\beta) \phi\left(\frac{1}{r_{i}^{+}}\right) \Delta^{+} E_{i-1}^{-1}\right]
\end{aligned} \\
& r_{i}^{+}=\frac{\left\langle\Delta^{+} E_{i}^{+}, \Delta^{+} E_{i-1}^{+}\right\rangle}{\left\langle\Delta^{+} E_{i-1}^{+}, \Delta^{+} E_{i-1}^{+}\right\rangle} \\
& r_{i}^{-}=\frac{\left\langle\Delta^{+} E_{i-1}^{-}, \Delta^{+} E_{i}\right\rangle}{\left\langle\Delta^{+} E_{i}^{-}, \Delta^{+} E_{i}^{-}\right\rangle}
\end{aligned}
$$


where $\mathrm{E}$ is the total flux, $\mathrm{E}^{+}$and $\mathrm{E}^{-}$are the split fluxes, $\Delta^{+}$is the forward differencing operator, and $\phi$ is a limiter. In Eqs. (8) and (9), the symbol $<>$ denotes the inner product. The expression of $\mathrm{E}_{\mathrm{i} \frac{1}{2}}$ is obtained by replacing $\mathrm{i}$ with $\mathrm{i}-1$ in the above equations.

Now comes the choice of the limiter and the flux splitting scheme. In the present work, Roe's "superbee" [3] and flux-difference splitting [4] are chosen. Such a preference is based on our experience with several Euler and Navier-Stokes solutions. Nevertheless, it should be pointed out that no limiter has been found to be universally satisfactory, and a drawback of Roe's flux-difference splitting is that it may not spread the expansion wave correctly.

\section{Unsteady Shock Tube Problems}

Numerical results for four different shock tube cases have been obtained by solving the 2-D Euler equations and compared with the exact 1-D solutions. Table 1 gives the normalized initial conditions of these standard test cases. For Sod's and Lax's problems, the computational domain is covered by $101 \times 21$ uniformly distributed grid points $(\Delta x=\Delta y=0.1)$. For the strong shock and large temperature ratio problems, $201 \mathrm{x} 41$ points $(\Delta \mathrm{x}=\Delta \mathrm{y}=0.05)$ are used. At the upstream as well as the downstream boundaries, flow quantities are consistently over specified by invoking the exact solutions. Along the top and bottom boundaries, symmetry conditions are imposed. The one-dimensionality of the calculated results has been confirmed, and the results along the horizontal centerline of the computational domain will be presented.

The Sod and Lax problems involve only moderate strength shock. For the Sod problem, constant $\Delta t=0.03$ is used to advance the solution over a period of 60 time steps. Results for values of $\beta$ between 0 and 1 have been obtained and 
compared well with the exact solution. As an example, the solutions obtained with $\beta=1.0$ are shown in Fig. 1. For the Lax problem, constant $\Delta t=0.017$ is used to advance the solution over a period of 85 time steps. Again, various $\beta$ values have been used and typical results for $\beta=1.0$ are presented in Fig. 2. For the large temperature ratio problem, fine grids and constant $\Delta t=0.0051$ are used. In this case, satisfactory results can only be obtained with $\beta \geq 0.75$. The results shown in Fig. 3 are obtained with $\beta=1.0$ over a period of 100 time steps. There are some oscillations in the neighborhood of shock and contact discontinuity.

As mentioned before, an apparent drawback of Roe's flux-difference splitting is that it may not spread the expansion wave correctly and thus lead to a nonphysical 'expansion shock' appearing in the computed flow. Fig. 4 illustrates such a case. For this shock tube flow, the pressure ratio is 30 , and the density ratio is 24 . The grid spacings are $\Delta \mathrm{x}=\Delta \mathrm{y}=0.05$, and a constant $\Delta \mathrm{t}=0.01$ is used to advance the solution over a period of 90 time steps. The comparison between the exact solution and the computed results obtained from the current TVD scheme with $\beta=1$ (i.e., the third-order-biased upwind scheme) indicates the appearance of an 'expansion shock' at $x=0$. It is noted here that the same 'expansion shock' also occurs for other values of $\beta$. When the pressure ratio is increased to the present strong shock level of 500 , the solution procedure quickly diverges as a consequence of a much stronger, nonphysical, expansion shock. One way to fix this problem is to add dissipation proportional to the strength of the expansion (see e.g. [3]). Under the current effort, the fourth-difference constant-coefficient artificial dissipation model [5] is adopted for this purpose. Fig. 5 illustrates the solutions obtained with $\beta=1.0$ and $\sigma=1.5$, where $\sigma$ is the value of the dissipation coefficient. As it can be seen, the calculated results exhibit some oscillations near the expansion head. 


\section{Inviscid Oblique Shock Reflection on a Plane Wall}

Numerical solutions of a regular shock reflection with an incident shock angle of 29 degrees and free stream Mach number of 2.9 have been obtained by solving the 2D Euler equations. The calculations are started by assuming uniform flow with $M_{\infty}=2.9$ everywhere except the top boundary, where the variables are consistently over specified from the jump conditions. The computational domain extends from $\mathrm{x}=0$ to $\mathrm{x}=4$ and from $\mathrm{y}=0$ to $\mathrm{y}=1$. Uniform grids are used to discretize the domain. Both coarse grid and fine grid calculations have been performed for the same CFL number. Table 2 lists the cases tested in this study. First of all, $\beta \geq 0.5$ seems to be a necessary condition for obtaining a converged solution. Secondly, the observation that, when $\beta=1.0$, the coarse grid calculation without the use of flux limiter manages to reach a converged albeit smeared solution while its counter part fine grid calculation can not yield a converged solution, simply indicates that sharpened shock profile requires the use of flux limiter to maintain its accuracy and numerical stability. Thus, it is concluded that robust calculation of shocked flow requires the use of flux limiter in conjunction with a value of $\beta \geq 0.5$. The pressure contours obtained from $122 \times 42$ grid points and with $\beta=1.0$ are shown in Fig. 6(a). The comparison of computed and exact solutions along $\mathrm{y}=0.4878$ is illustrated in Fig. 6(b) and 6(c). Good results along other $\mathrm{y}=$ constant lines are also obtained.

5. Supersonic Boundary Layer Flow Over a Flat Plate

A Mach 2.2 laminar flow over an adiabatic flat plate is calculated by solving the 2D Navier-Stokes equations. The Reynolds number based on the free stream condition and a reference length of $0.08 \mathrm{~m}$ is $9.8645 \times 10^{4}$. In the streamwise direction $(-0.19 \leq x \leq 2.00$ with $x=0$ being the leading edge of the plate), 74 grid points 
with a constant $\Delta x=0.03$ are used. In the direction normal to the wall $(0 \leq y \leq 1.19212)$, 62 grid points with the following distribution are used.

$$
\begin{aligned}
\Delta y_{j}=1.5625 \times 10^{-4} & , & 1 \leq j \leq 4, \\
\Delta y_{j}=1.1868 \Delta y_{j-1} & , & 5 \leq j \leq 33 \\
\Delta y_{j}=3.75 \times 10^{-2} & , & j \leq 33 .
\end{aligned}
$$

Several calculations have been performed to investigate the effects of $\beta$ and the use of flux-limiter on the calculated boundary layer properties. All the calculations are carried out with a constant dimensionless time step $\Delta t=0.01$. Table 3 shows the parameters of these cases and a short description of the most important observation. These results suggest that the use of flux-limiter does not degrade the computed boundary layer flow. Furthermore, the present Navier-Stokes solutions compare quite well with the corresponding solutions obtained by executing an existing 2D compressible boundary layer code [6], as illustrated in Fig. 7. These results are obtained with $\beta=1.0$ and limiter on. For the evaluation of boundary layer properties such as the displacement and momentum thicknesses, the boundary layer edge is assumed to be at a point where the u-velocity is $99.5 \%$ of its free stream values. The profiles of $u$-velocity and temperature are normalized with free stream velocity and temperature respectively. They are then plotted against $\mathrm{y} / \theta$ where $\theta$ denotes the local momentum thickness. The skin friction coefficient is evaluated by using the first order backward differencing, and then plotted against $\mathrm{Re}_{\mathbf{X}}$, which is the Reynolds number based on the local distance from the leading edge of the plate. 
6. Interaction of Oblique Shock Wave and Laminar Boundary Layer.

The main features of the oblique shock wave and laminar boundary layer interaction are well understood. A brief description of these features is given in Fig. 8. Since this configuration involves shocks and separated flow, and carries no uncertainty associated with turbulence modeling, it is chosen here as a test case. The numerical solutions at the asymptotic steady state are compared with the experimental data reported in Ref. [7]. In addition, time-accurate calculations are performed to illustrate the transient development of this interaction.

The flow parameters are such that $M_{\infty}=2.2, R e=\rho_{\infty} \mathrm{u}_{\infty} \mathrm{x}_{\mathrm{sh}} / \mu_{\infty}=9.8645 \times 10^{4}$, where $\mathrm{X}_{\mathrm{Sh}}=0.08 \mathrm{~m}$ is the distance between the leading edge and shock impingement point in the experiment. In addition, the shock incident angle is 30.027 degrees. Based on the studies discussed in Sections 4 and 5, the third-order-biased upwind (i.e., $\beta=1$ ) TVD scheme is used for the present calculations. The total number of grid points is $74 \times 62$, and they are distributed in two different ways, as depicted in Fig. 9. For a given grid distribution, a steady-state boundary layer flow is first established. Then, an oblique shock wave is imposed at the upper left corner of the inflow boundary and along the top boundary, where the variables are consistently over specified from the jump conditions. The subsequent transient development of the interacting process is followed by employing $\Delta t=0.01$, until an asymptotic steadystate is reached.

The grid distribution denoted by 'initial grids' is deduced from the triple-deck theory as described by Eq. (9) together with a constant $\Delta x=0.03$. The grid distribution denoted by 'adapted grids' is constructed by using the grid adaptation package TURBOAD [8]. More specifically, a steady state solution of the interaction is first obtained by using the initial grids. Using this grid distribution as a baseline and taking into account the large gradient regions in the flow solution, TURBOAD 
enables a redistribution of grid points to improve the resolution in the shock and recirculation regions. Such an improvement is demonstrated in Fig. 10(a), which compares the measured wall pressure data and several computed results obtained from different grid distributions. It is noted here that, in Fig. 10(a), the pressure is normalized by Po, i.e., the minimum pressure just upstream of the interaction. The calculated skin friction distribution is shown in Fig. 10(b). Separation and reattachment points can be determined from the positions where the skin friction vanishes. Table 4 gives the locations of these points as determined from computations and experiment. The values determined from the adapted grids are in close agreement with the experimental values reported in Ref. [7]. Fig. 11 shows the measured and computed streamwise velocity profiles in the separated region. Since the results obtained from the adapted grids require further two-dimensional interpolations between grid points, the profiles obtained from the initial grid distribution are used for convenience. It is also noted here that, as discussed in Ref. [7], there are errors in the experimental velocity measurements due to the behavior of the seeding particles.

The transient development of the interaction process is illustrated by the computed flow fields at three time stations, i.e., at the moment of shock impingement ( $\mathrm{t}=3.3 \times 10^{-4}$ seconds), the subsequent interaction in the upstream and downstream region $\left(t=5.4 \times 10^{-4}\right.$ seconds), and the final asymptotic steady state. Figures 12 and 14(a), (b) describe the development of shock reflection and expansion fan in terms of the static pressure contours and wall pressure distribution (normalized by $P_{\infty}$ ). Figures 13 and 14(c), (d) illustrate the emergence of a separation region and the thickening of the boundary layer in terms of the Mach number contours and skin friction distributions. It is noted here that the wall pressure and skin friction distributions are plotted in the computational space, i.e., the abscissa I is the streamwise grid index, and these results are obtained from the 
adapted grid distribution. These figures, together with many others at different time stations, indicate that the entire process is essentially monotonic, i.e., no appreciable oscillation in flow patterns has been observed.

\section{Concluding Remarks}

Under the present effort, a TVD scheme has been developed and incorporated into an existing time-accurate high-resolution Navier-Stokes code. Test cases have been run to assess the overall accuracy and the robustness of the developed solution procedure. These numerical results compare well with corresponding analytic solution or experimental data. Its capability of accurately tracking transient movement of shock waves and contact discontinuities is demonstrated by calculating the shock tube problems. Its accuracy in calculating multi-dimensional shock waves and boundary layer flows is demonstrated by solving inviscid regular shock reflection and a supersonic boundary layer. It is then applied to compute the interaction of an oblique shock wave with a flat plate boundary layer, both the transient development and the asymptotic steady state of the interaction are presented to add some insight into this classical problem. 
References

1. Chen, S.-C., Liu, N.-S., and Kim, H.D., "An Iterative Implicit Diagonally-

Dominant Factorization Algorithm for Solving the Navier-Stokes Equations," NASA TM 105259, October 1991

2. Liou, M.-S., "A Generalized Procedure for Constructing an Upwind-Based TVD Scheme," AIAA paper 87-0355, 1987.

3. Roe, P.L., "Some Contributions to The Modeling of Discontinuous Flows." Lectures on Appl. Math. 22(1985), Part II, pp. 163-194.

4. Roe, P.L., "Approximate Riemann Solvers, Parameter Vectors, and Difference Schemes," J. Comput. Phys. Vol. 43, pp. 357-372 (1981).

5. Steger, J.L., "Implicit Finite-Difference Simulation of Flow about Arbitrary TwoDimensional Geometries," AIAA Journal, Vol. 16, No. 7, pp. 679-686 (1978).

6. Harris, J.E. and Blanchard, D.K., "Computer Program for Solving Laminar, Transitional, or Turbulent Compressible Boundary-Layer Equations for TwoDimensional and Axisymmetric Flow," NASA TM 83207, February 1982.

7. Degrez, G., Boccadoro, C.H., and Wendt, J.F., "The Interaction of An Oblique Shock Wave with A Laminar Boundary Layer Revisited. An Experimental and Numerical Study," J. Fluid Mech., 177(1987), pp. 247-263.

8. Henderson, T.L., Choo, Y.K., and Lee, K.D., "Interactive Solution-Adaptive Grid Generation Procedure," NASA TM 105432, April 1992. 


\begin{tabular}{|c|c|c|c|c|c|c|}
\hline & \multicolumn{3}{|c|}{$0<\mathrm{x}<5$} & \multicolumn{3}{c|}{$5<\mathrm{x}<10$} \\
\cline { 2 - 7 } & $\rho$ & $\mathrm{u}$ & $\mathrm{p}$ & $\rho$ & $\mathrm{u}$ & $\mathrm{p}$ \\
\hline Sod's Problem & 8 & 0 & 10 & 1 & 0 & 1 \\
\hline Lax's Problem & 0.890 & 0.698 & 6.179 & 1 & 0 & 1 \\
\hline $\begin{array}{c}\text { Large Temperature } \\
\text { Ratio Problem }\end{array}$ & $10 / 3$ & 0 & 100 & 1 & 0 & 1 \\
\hline Strong Shock & 400 & 0 & 500 & 1 & 0 & 1 \\
Problem & & & & & & \\
\hline
\end{tabular}

Table1. Normalized Initial Conditions for Shock Tube Cases

\begin{tabular}{|c|c|c|c|}
\hline & $\beta$ & limiter & Remark \\
\hline \multirow[t]{9}{*}{ Coarse Grids $(61 \times 21)$} & 0.00 & on & No solution \\
\hline & 0.10 & on & No solution \\
\hline & 0.25 & on & \\
\hline & 0.50 & on & \\
\hline & 1.00 & on & \\
\hline & 0.00 & off & No solution \\
\hline & 0.25 & off & No solution \\
\hline & 0.50 & off & \\
\hline & 1.00 & off & \\
\hline \multirow[t]{4}{*}{ Fine Grids $(122 \times 42)$} & 0.25 & on & No solution \\
\hline & 0.50 & on & \\
\hline & 1.00 & on & \\
\hline & 1.00 & off & No solution \\
\hline
\end{tabular}

Table 2. Cases Studied for Oblique Shock Wave Reflection in Inviscid Flow 


\begin{tabular}{|c|c|c|}
\hline$\beta$ & limiter & Remark \\
\hline 0.0 & off & $\begin{array}{c}\text { significant spatial oscillations in boundary layer } \\
\text { thickness and skin friction }\end{array}$ \\
\hline 0.5 & off & $\begin{array}{l}\text { no appreciable differences from } \beta=1 \text { and limiter-on } \\
\text { case }\end{array}$ \\
\hline 0.5 & on & $\begin{array}{l}\text { essentially the same as } \beta=0.5 \text { and limiter-off case } \\
\text { except for some spatial oscillations in boundary layer } \\
\text { thickness }\end{array}$ \\
\hline 1.0 & off & $\begin{array}{l}\text { slightly higher wall temperature than the } \beta=1 \text {, } \\
\text { limiter-on case }\end{array}$ \\
\hline 1.0 & on & Results shown in Fig. 7 \\
\hline
\end{tabular}

Table 3. Cases Studied for Supersonic Laminar Boundary Layer Flow

\begin{tabular}{|c|c|c|}
\hline & Separation (X/Xsh) & Reattachment (X/Xsh) \\
\hline Present code (initial grids) & 0.745 & 1.390 \\
\hline Present code (adapted grids) & 0.795 & 1.256 \\
\hline Degrez' experiment & 0.78 & 1.28 \\
\hline Degrez' computation (M=2.15) & 0.79 & 1.24 \\
\hline
\end{tabular}

Table 4. Separation and Reattachment Points in Shock Boundary Layer Interaction 


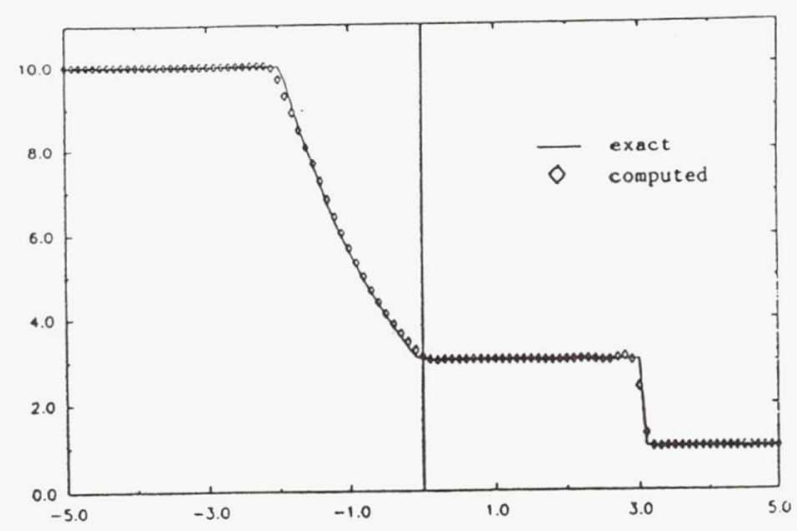

(a) pressure

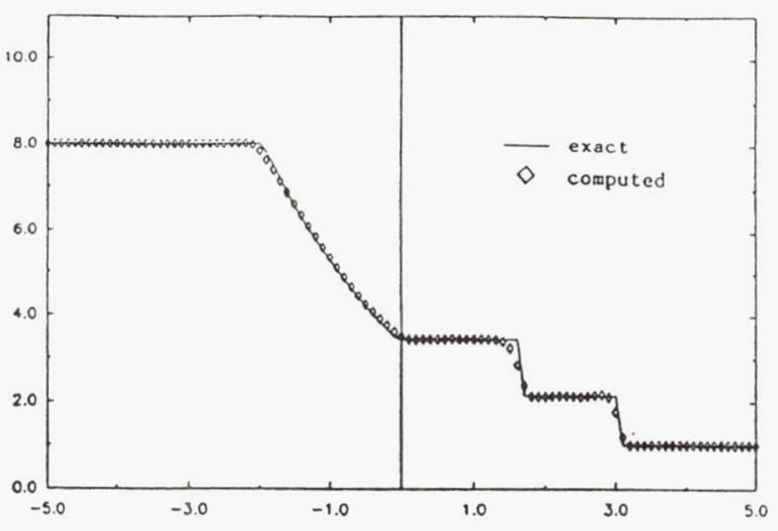

(b) density

Fig. 1. Sod's problem $(\beta=1.0)$

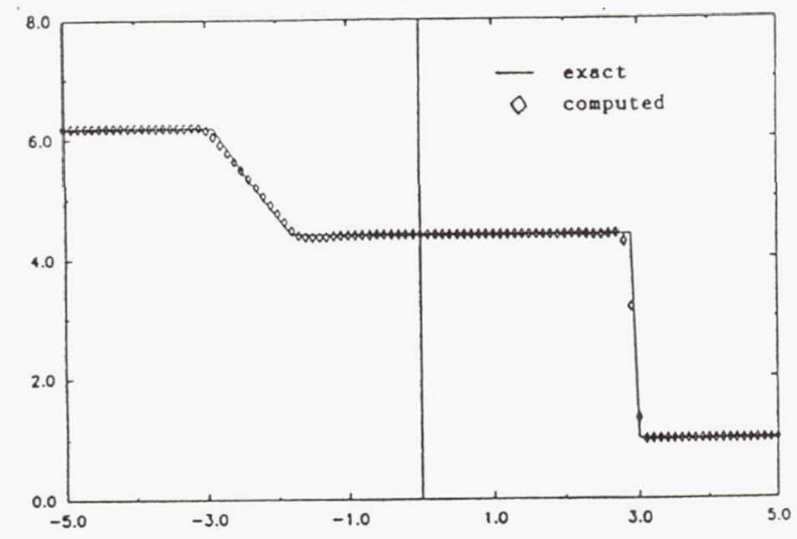

(a) pressure

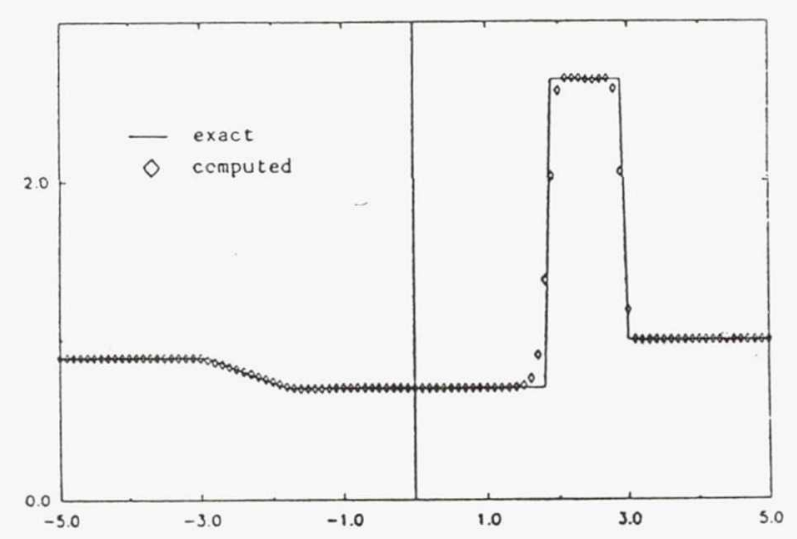

(b) density

Fig. 2. Lax's problem $(\beta=1.0)$

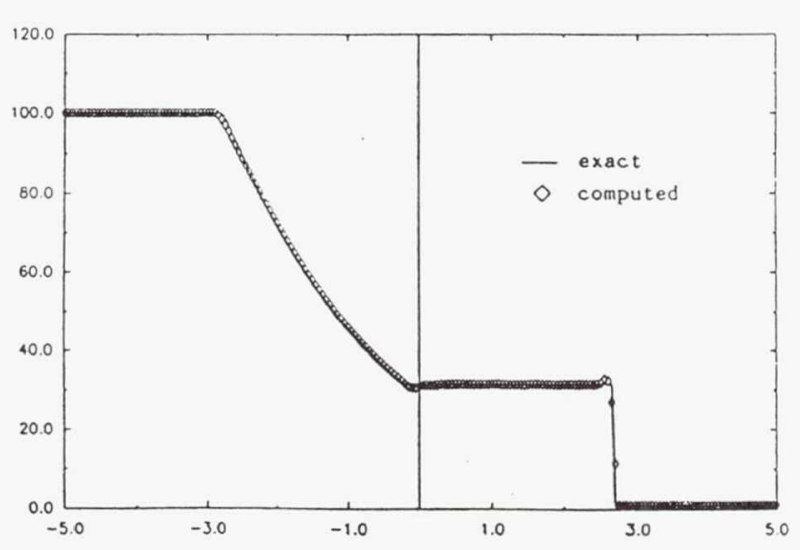

(a) pressure

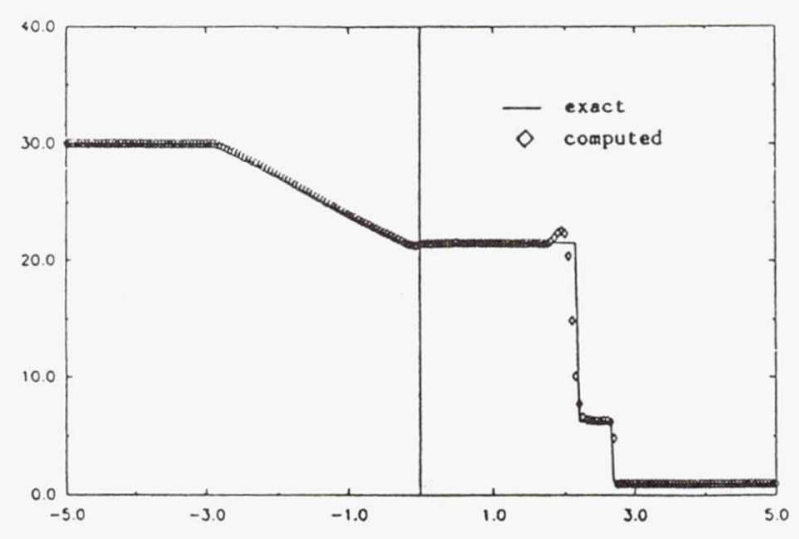

(b) temperature

Fig. 3. Large temperature ratio problem $(\beta=1.0)$ 


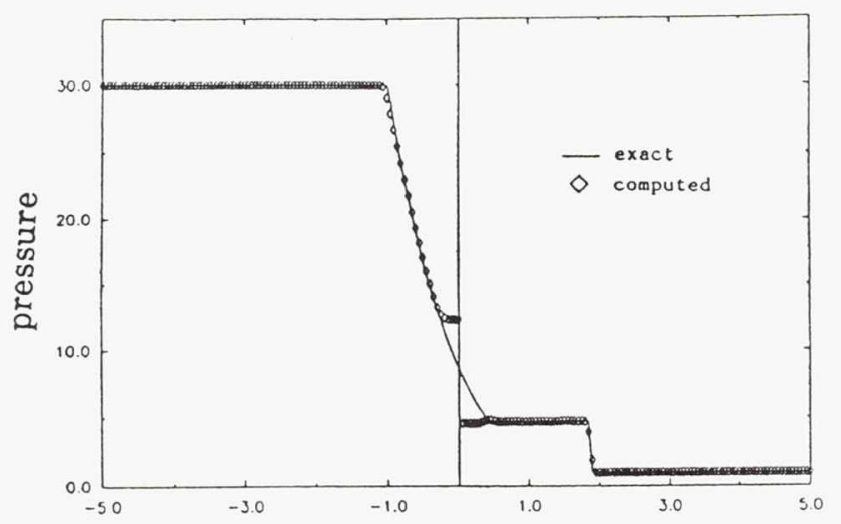

Fig. 4. The appearance of expansion shock in computed flow $(\beta=1.0$ and $\sigma=0.0)$

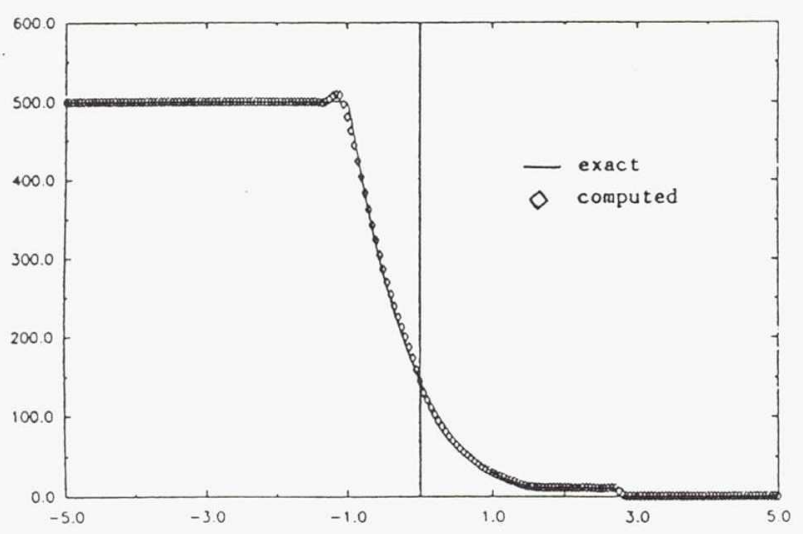

(a) pressure

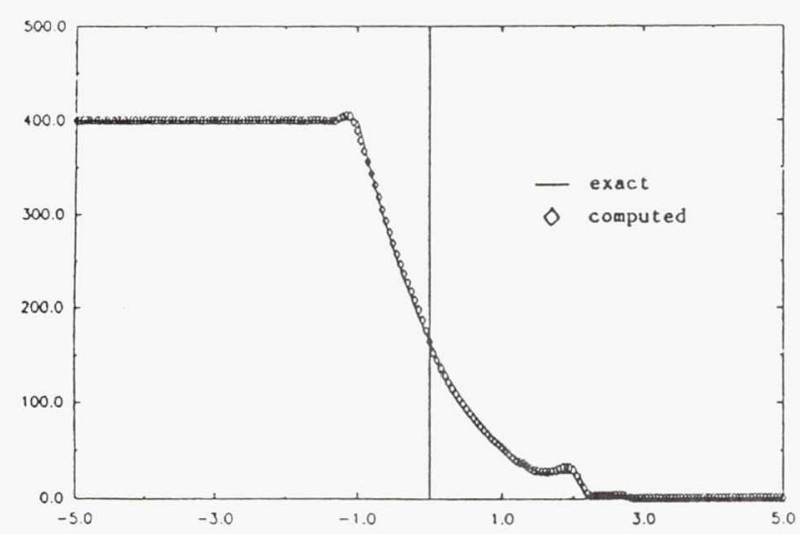

(b) density

Fig. 5. Strong shock problem $(\beta=1.0$ and $\sigma=1.5)$ 


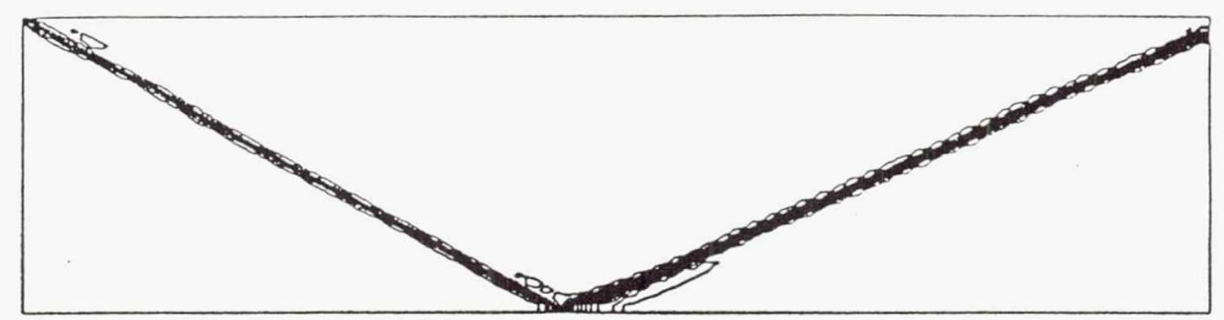

(a) pressure contours

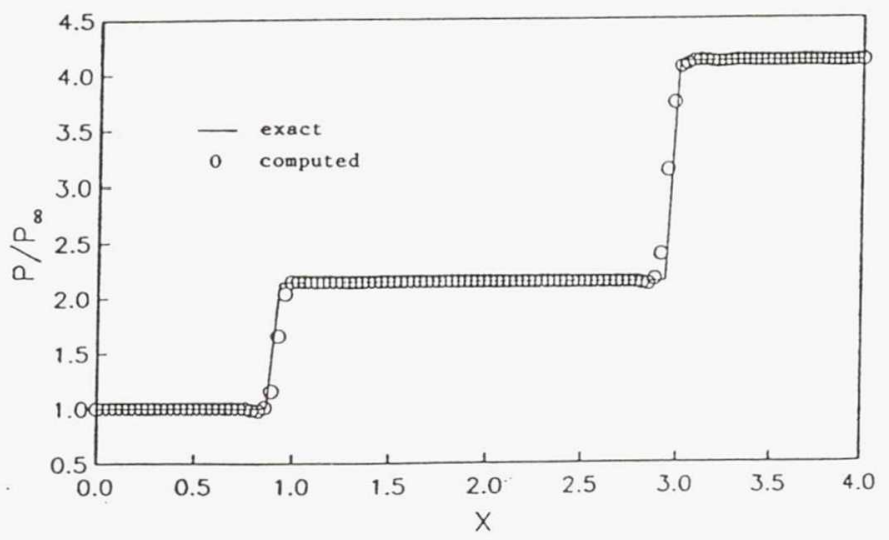

(b) pressure distribution ( $\mathrm{y}=0.4878)$

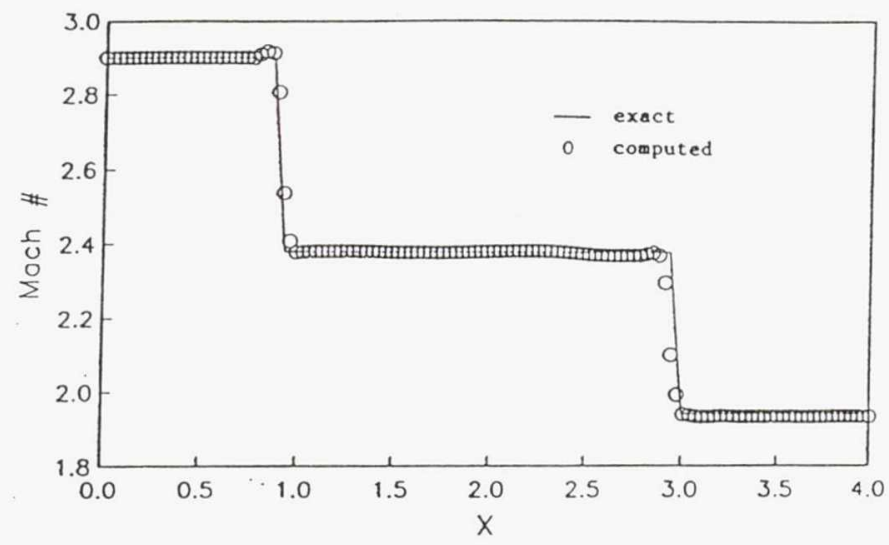

(c) mach number distribution $(\mathrm{y}=0.4878)$

Fig. 6. Oblique shock reflection in inviscid flow $\left(\mathrm{M}_{\infty}=2.9\right.$, incoming shock angle $\left.=29^{\circ}, \beta=1.0\right)$ 


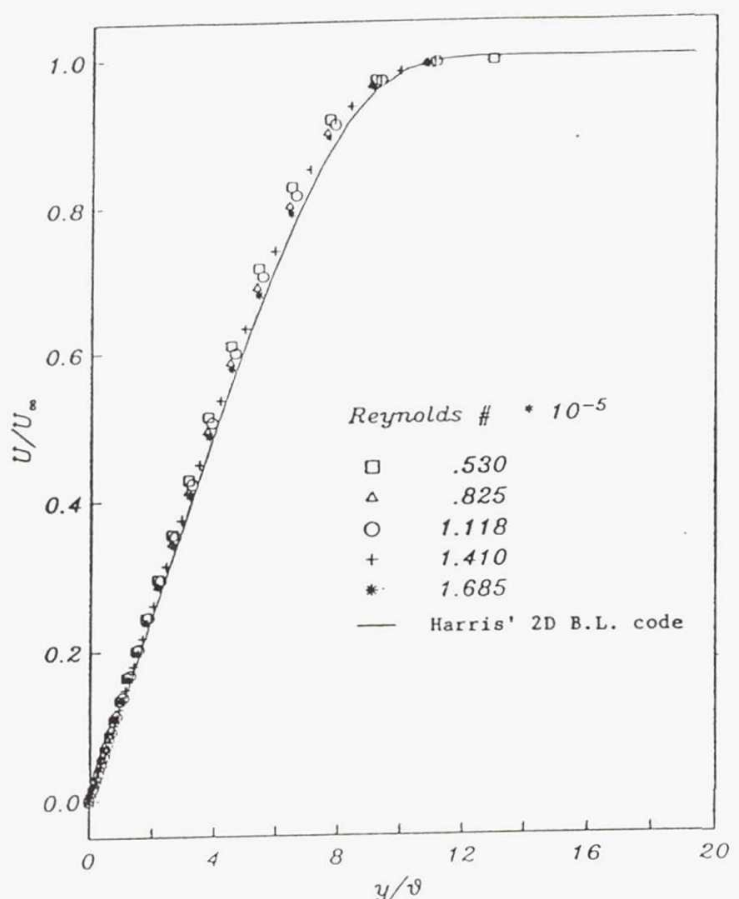

(a)

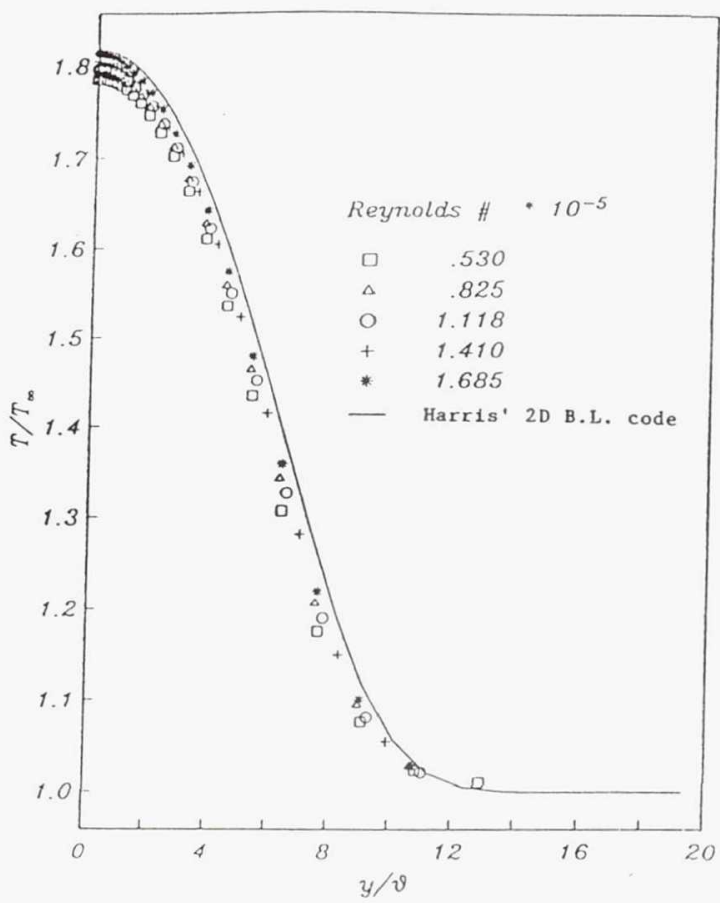

(b)

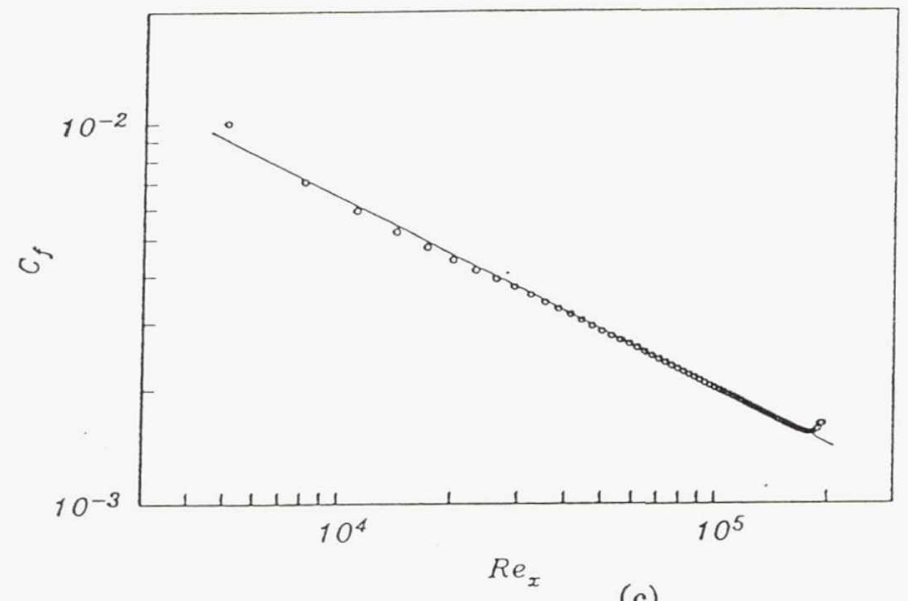

- present code

- Harris' 2D B.L. code

(c)

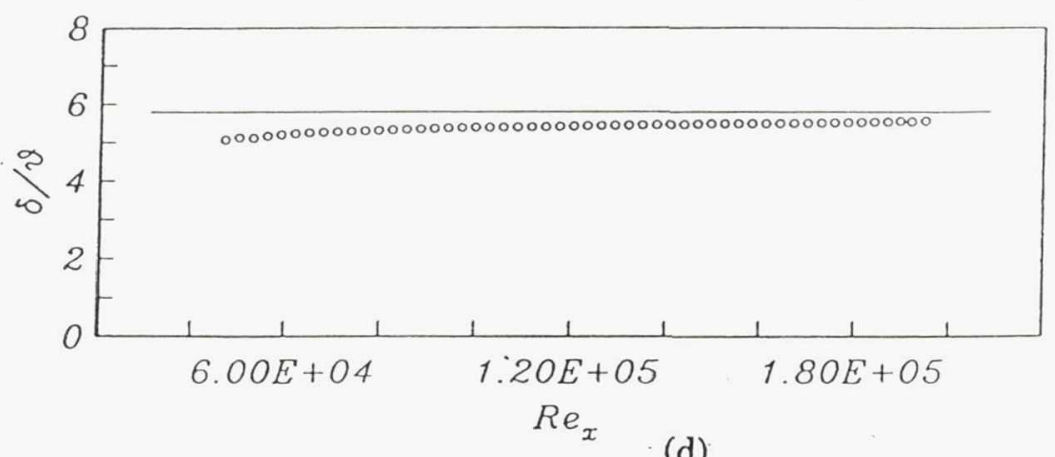

- present code

- Harris' 2D B.L. code

Fig. 7. Solutions for the supersonic laminar boundary layer over a flat plate ( $\beta=1.0$ and limiter on) (a) normalized u-velocity profiles (b) normalized temperature profiles (c) skin friction coefficient (d) ratio of displacement thickness to momentum thickness 
Page intentionally left blank 

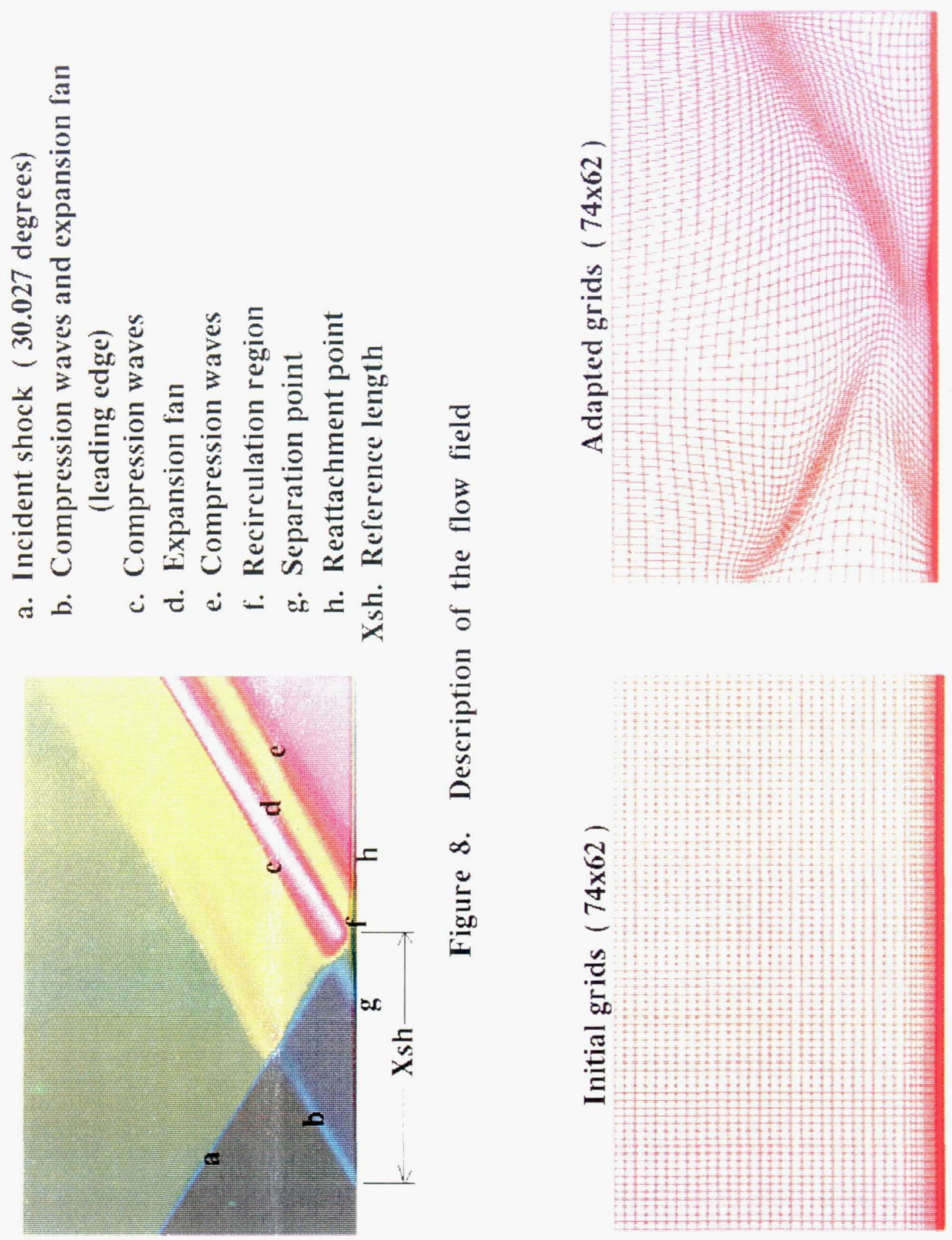

泀

尝 
Page intentionally left blank 

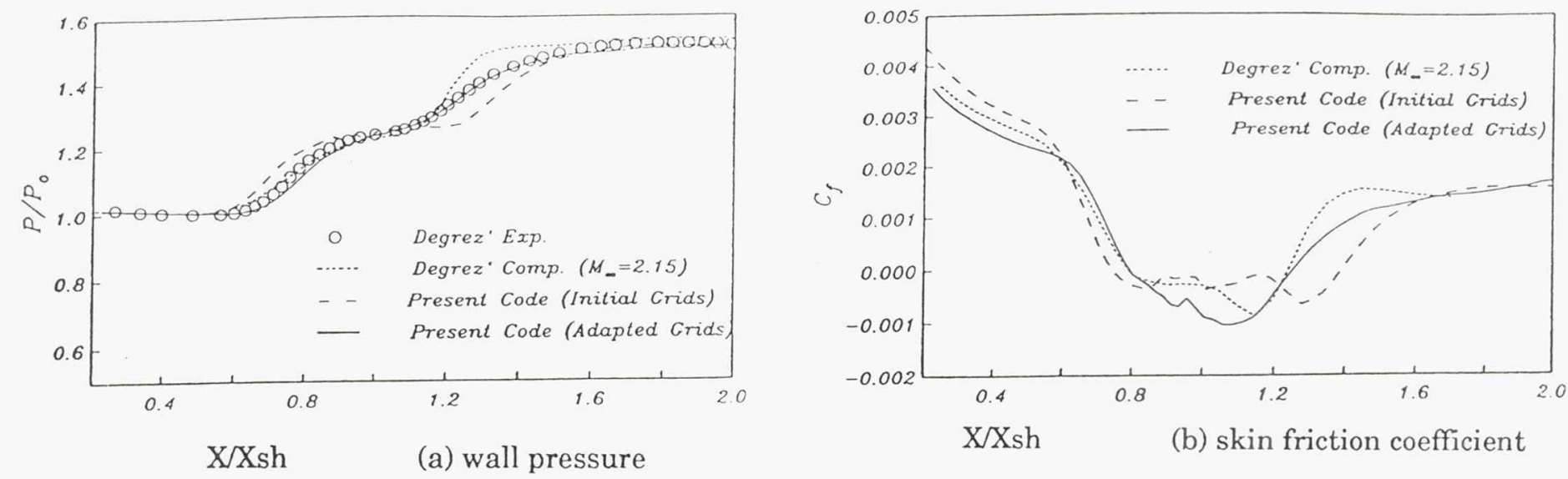

Fig. 10. Shock boundary layer interaction

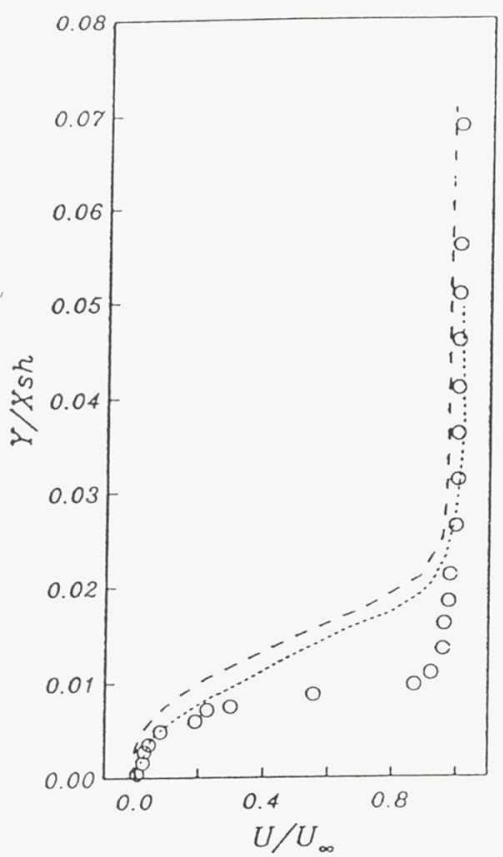

(a)

\footnotetext{
- Degrez'Exp.

Degrez' Comp. $\left(M_{-}=2.15\right)$

$X / X$ sh $=0.80$

- Present Code (Initial Crids) $X / X \operatorname{sh}=0.79$
}

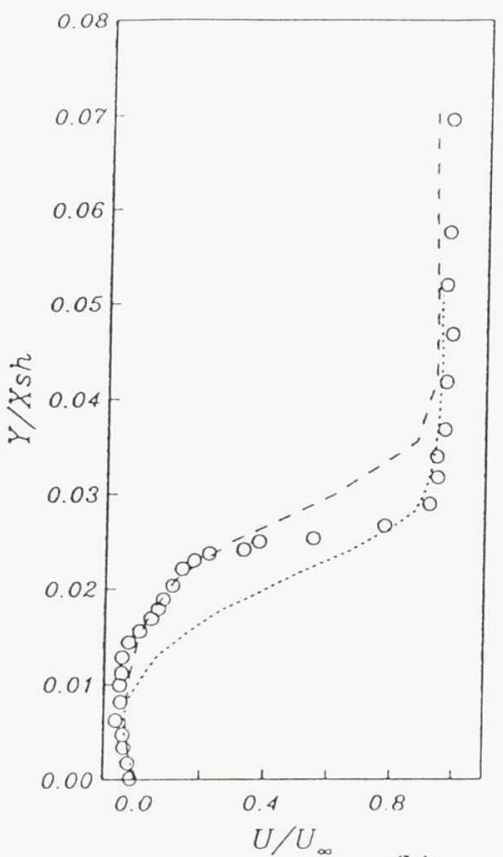

(b)

$$
\begin{aligned}
& \text { Degrez' Exp. } \\
& \left.\cdots \text { Degrez' Comp. (M... } \quad M_{\infty}=2.15\right) \\
& X / X \text { sh }=1.00 \\
& \\
& -\quad \text { Present Code (Initial Grids) } \\
& X / X \operatorname{sh}=1.00
\end{aligned}
$$

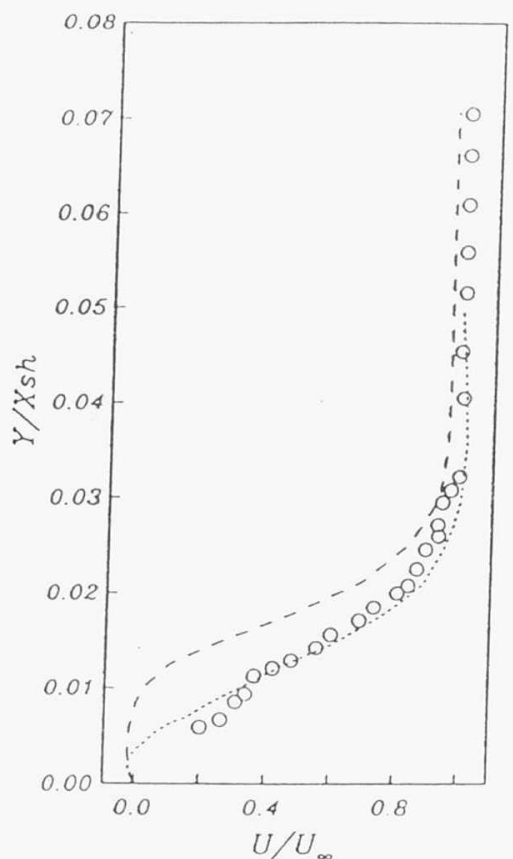

(c)

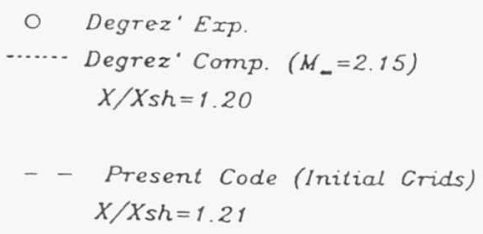

Fig. 11. Streamwise velocity profiles (shock boundary layer interaction) 
Page intentionally left blank 


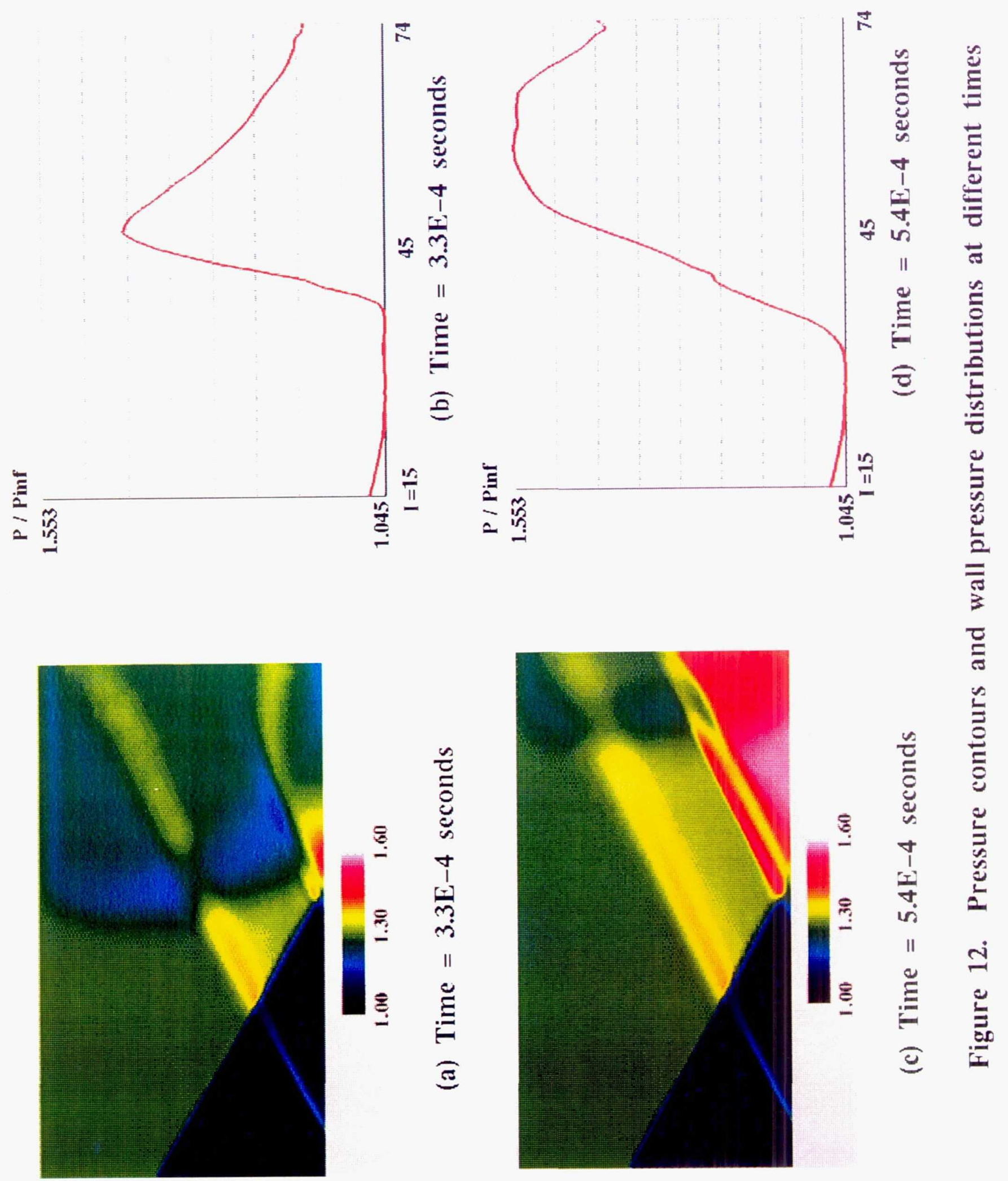


Page intentionally left blank 


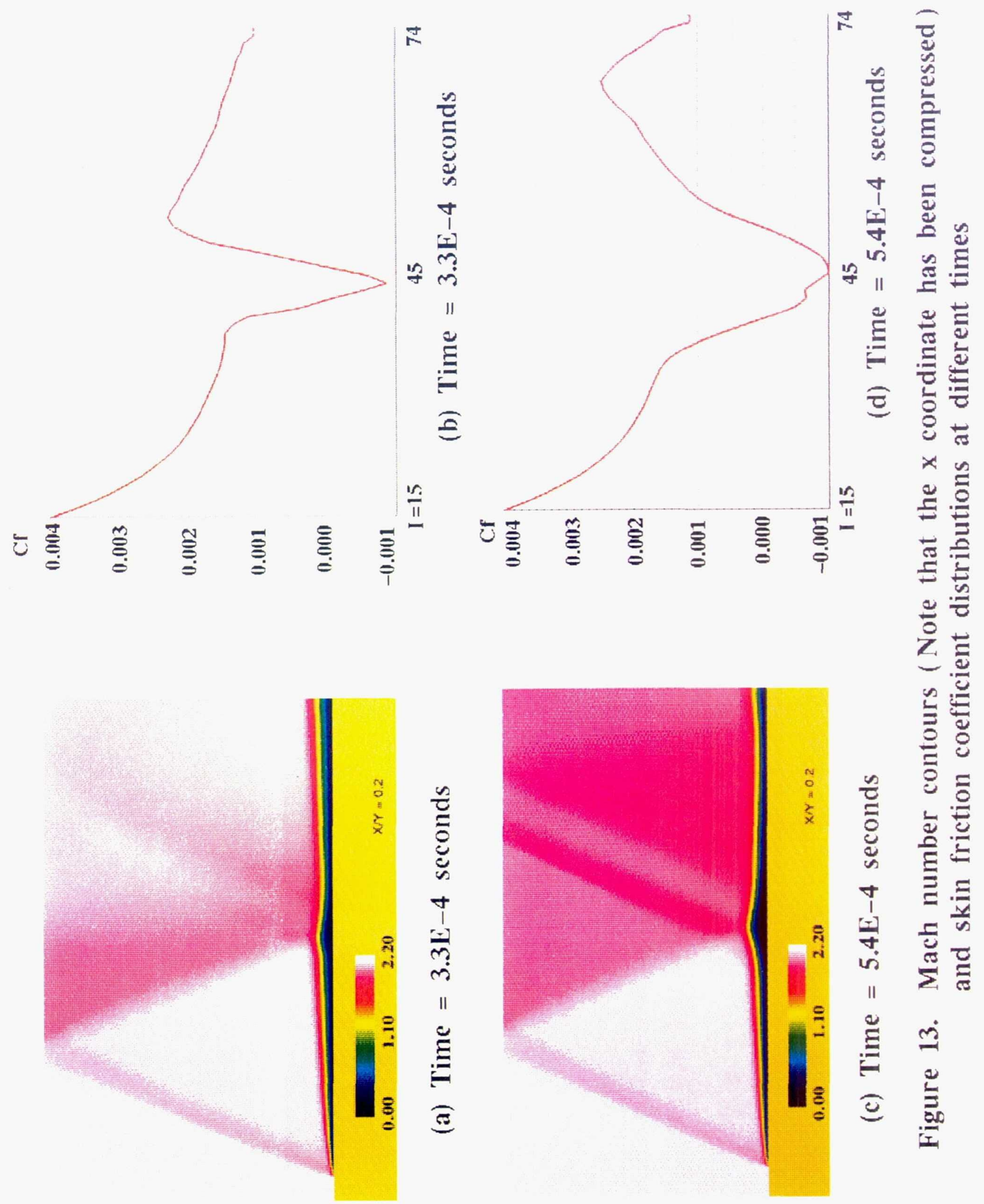


Page intentionally left blank 


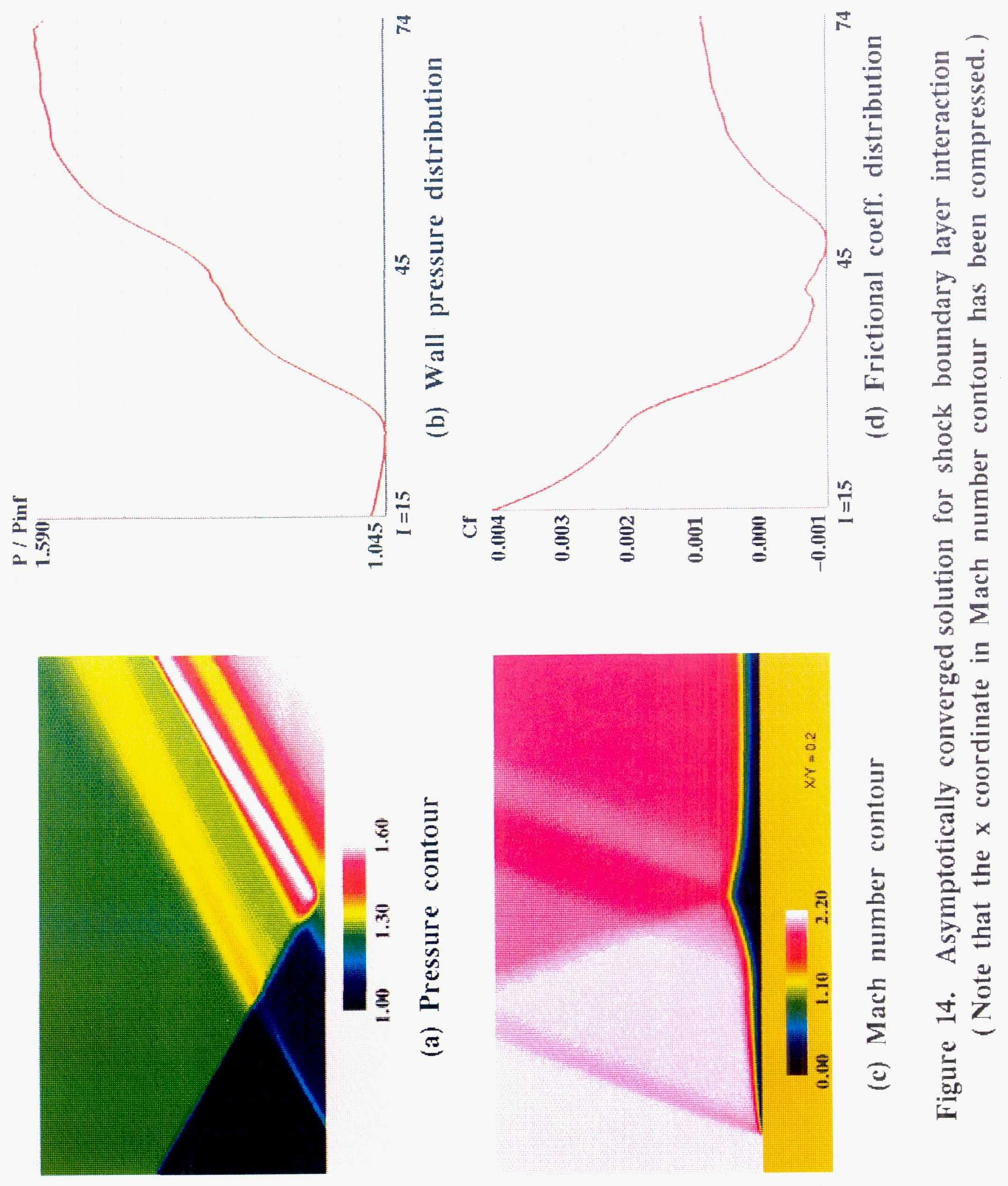




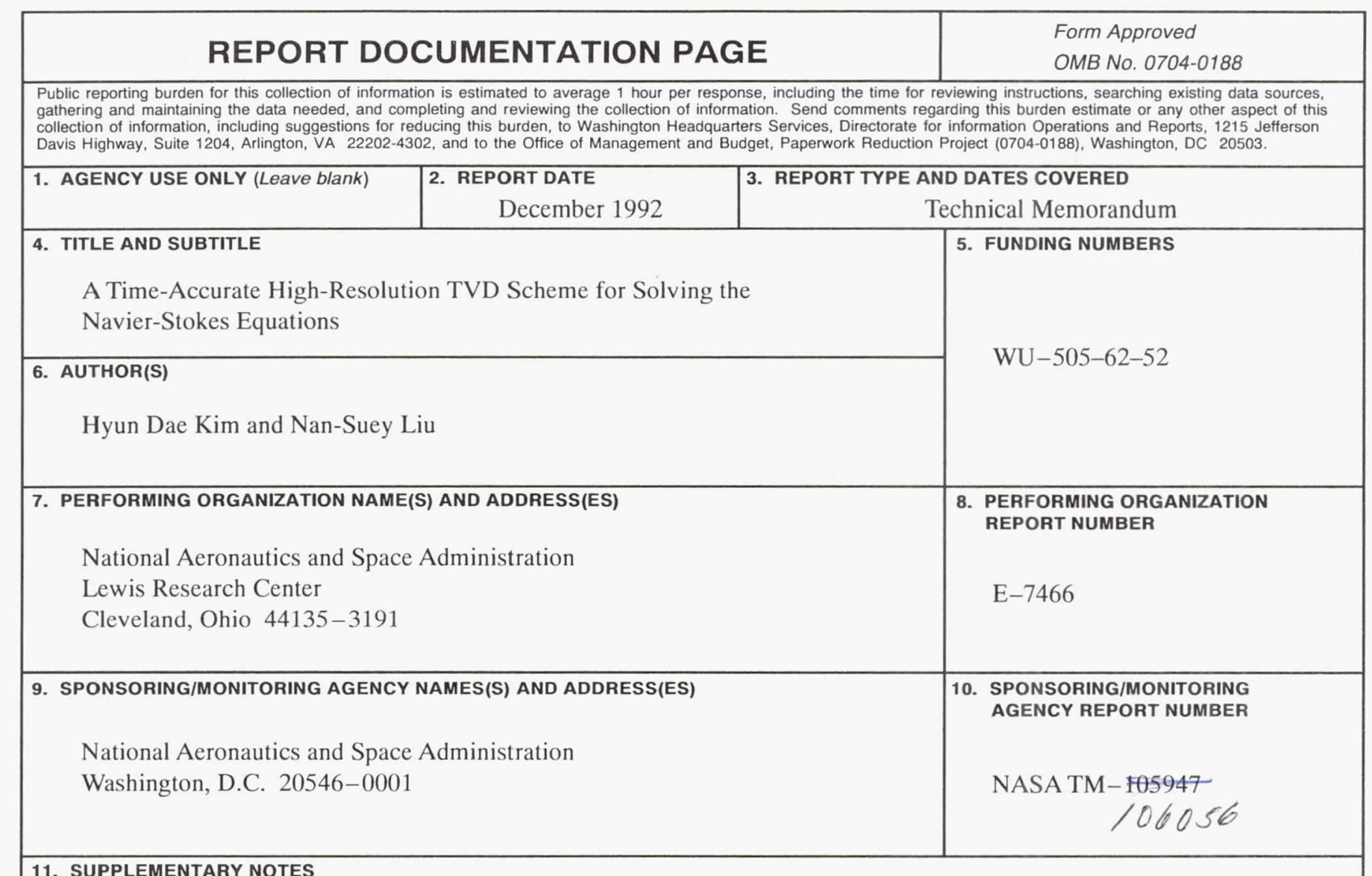

Hyun Dae Kim and Nan-Suey Liu, NASA Lewis Research Center, Cleveland, Ohio.

12a. DISTRIBUTION/AVAILABILITY STATEMENT

12b. DISTRIBUTION CODE

Unclassified - Unlimited

Subject Category 64

13. ABSTRACT (Maximum 200 words)

A TVD scheme has been developed and incorporated into an existing time-accurate high-resolution Navier-Stokes code. The accuracy and the robustness of the resulting solution procedure have been assessed by performing many calculations in four different areas: shock tube flows, regular shock reflection, supersonic boundary layer, and shock boundary layer interactions. These numerical results compare well with corresponding exact solutions or experimental data.

14. SUBJECT TERMS

Total variation diminishing scheme; Shock tube flow; Boundary layer; Oblique shock wave reflection; Shock boundary layer interaction

\begin{tabular}{|c|c|}
\hline $\begin{array}{c}\text { 17. SECURITY CLASSIFICATION } \\
\text { OF REPORT } \\
\text { Unclassified }\end{array}$ & $\begin{array}{c}\text { 18. SECURITY CLASSIFICATION } \\
\text { OF THIS PAGE } \\
\text { Unclassified }\end{array}$ \\
\hline
\end{tabular}

19. SECURITY CLASSIFICATION OF ABSTRACT Unclassified 
National Aeronautics and Space Administration

Lewis Research Center

Cleveland, Ohio 44135

Official Business

Penalty for Private Use $\$ 300$
FOURTH CLASS MAIL

ADDRESS CORRECTION REQUESTED
|||||

USMAII

Postage and Fees Paid

National Aeronautirs an

Space Administration

NASA 451 